\section{Wie handelt das Hirn?}

Wir gewinnen zunehmend Erkenntnisse über die Komplexität unseres Gehirns. Prof. Dr. Chris Frith, Professor für Neuropsychologie am University College of London ist ein international anerkannter Vertreter dieses jungen wissenschaftlichen Zweiges. Leicht verständlich und spannend bringt er dem Leser die Funktionsweise des Gehirns im Austausch mit der Umwelt nahe. Sein Buch ist lesenswert für jeden, der wissenschaftlich fundiert über das Gehirn lernen will. Dabei legt er wegweisende Studien des letzten Jahrzehnts zugrunde und interpretiert sie. So etwa die Studie von Libets (1986), die implizierte, dass wir "keinen freien Willen haben". Libet konnte nachweisen, dass das Bereitschaftspotenzial, einen Finger zu heben, bereits 300 Millisekunden vor dem Wunsch, den Finger zu heben, auftritt. Aber das heißt nicht, wie vorschnell geschlussfolgert wurde, dass die Handlung nicht frei gewählt worden ist. Es bedeutet einfach nur, dass wir uns nicht bewusst sind, die Entscheidung zu dieser Handlung zu einem früheren Zeitpunkt getroffen zu haben.

Im zweiten Teil seines Buches geht der Autor darauf ein, wie das Gehirn zu seinen komplexen Leistungen fähig ist. Er erklärt anschaulich das Phänomen des assoziativen Lernens und berichtet über neuere Erkenntnisse im Bereich der Spiegelneurone. Er kommt zu dem Schluss, dass unsere Wahrnehmung der Welt eine Fantasie ist, die mit der Realität (meistens) im Einklang steht. Mir wurde beim Lesen des Buches klar, warum ich über mehrere Tage immer ins falsche Zimmer lief, nachdem wir die Funktion von zwei Praxiszimmern ausgetauscht hatten: Unser Gehirn bereitet automatisch Handlungsprogramme für die Objekte um uns herum vor. Es wird auch verständlich, warum ein Ereignis unterschiedlich von Beobachtern wahrgenommen und geschildert wird: Was wir wahrnehmen, ist ein von unserem Gehirn geschaffenes Modell - und abhängig von unseren Erfahrungen und Wahrnehmungen.

Dr. Kirsten Stollhoff

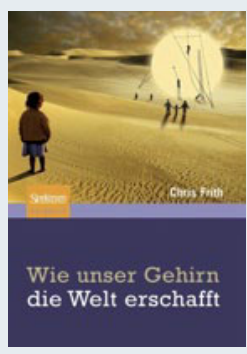

Chris Frith

Wie unser Gehirn die Welt erschafft

Spektrum Akademischer Verlag, Heidelberg 2010 $24,95 €$ ISBN 978-3-82742343-6 oder im Freundeskreis. Eine Abgrenzung von der Stimmbanddysfunktion (Vocal cord dysfunction) ist wichtig und erfolgt durch genaue Anamnese und endoskopische Untersuchung im Intervall. Eine hahnenkammartige exspiratorische Flussvolumenkurve kann Hinweis sein.

Die Therapie besteht aus verhaltenstherapeutischen Ansätzen, d.h. das nicht gewünschte Verhalten wird ignoriert, das gewünschte Verhalten gestärkt.

\section{Therapie}

Der Therapie des chronischen Hustens im Kindesalter sollte immer eine sorgfältige Diagnostik vorangehen, um schwere zugrunde liegende Erkrankungen nicht zu übersehen (Tab. 3).

Kinder mit viralen respiratorischen Infekten ohne Hinweis auf eine bakterielle Superinfektion in gutem Allgemeinzustand werden gewissenhaft beobachtet; meist verschwindet der Husten nach vier bis sechs Wochen von selbst. Rauchen sollte vermieden werden $[1,2]$.

Nicht verschreibungspflichtige Medikamente haben in kontrollierten Studien keine Besserung bei Kindern mit Schnupfen und Husten erbracht, im Gegenteil: Zentraldämpfende antitussive Medikamente können gerade bei kleinen Kindern ausgeprägte Nebenwirkungen (Atemdepression) zeigen [10].

Bei größeren Kindern mit isoliertem chronischem Husten v. a. nach viralen Infektionen kann ausnahmsweise vorübergehend nachts eine kurzzeitige antitussive Therapie erwogen werden. Codein ist der effektivste zentrale Hustenstiller, zeigt jedoch auch zentraldämpfende Effekte. Beim infektionsbedingten chronischen Husten gibt es keine Evidenz für mukolytische Medikamente wie Acetylcystein oder Bromhexidin; diese können eine weitere Irritation der Atemwege herbeiführen.

Bei Kindern mit chronischer Bronchitis und bereits bestehenden postinfektiösen Bronchiektasien ist außer einer sorgfältigen Physiotherapie mit Sekretmobilisation oft der frühzeitige Einsatz von Antibiotika für ca. vier Wochen hilfreich. Eine antiasthmatische Therapie mit inhalativen Steroiden oder Bronchodilatatoren sollte nur durchgeführt werden, wenn die Ursache des chronischen Hustens definitiv ein Asthma ist [12]. Bei V. a. auf

\section{Fazit für die Praxis}

- Bei einem chronischen Husten handelt es sich meist um eine Aneinanderreihung von viralen Infektionen der Atemwege, oft begünstigt durch verschiedene Prädispositionsfaktoren.

_Bei Kindern mit viralen Infektionen ohne Hinweis auf eine bakterielle Superinfektion verschwindet der Husten meist nach vier bis sechs Wochen von selbst.

Fremdkörperaspiration ist die bronchoskopische Untersuchung und direkte Entfernung des Fremdkörpers Therapie der Wahl. Insbesondere bei chronischem Verlauf ist eine perioperative antibiotische Therapie sinnvoll.

Ein gastroösophagealer Reflux kann sowohl medikamentös als auch operativ angegangen werden. Besteht der Husten als einzige klinische Auffälligkeit, ist eine Operation selten notwendig.

Die meisten kongenitalen Fehlbildungen sind möglichst operativ zu versorgen. Bei Hämangiomen genügt meist die Verödung $\left(\mathrm{CO}_{2}\right.$-Laser $)$, was jedoch oft mehrfach durchgeführt werden muss.

Ösophagotracheale Fisteln oder Larynxspalten sind operativ zu versorgen.

Die ausschlaggebende Therapie bei der primären ziliären Dyskinesie ist die Physiotherapie, die prompte antibiotische Therapie bei Infekten sowie eine Inhalationstherapie mit Beta-2-Mimetika.

Bei psychogenem Husten ist eine verhaltenstherapeutische Abklärung und Therapie notwendig. Antitussive Medikamente sind nur in seltenen Fällen indiziert.

\section{Literatur}

Der Beitrag inklusive Literatur ist als PDF-Datei unter www.springermedizin.de/paediatriehautnah abrufbar.

\section{Dr. med. Felicitas Nagel}

Kinderklinik und Poliklinik im

Dr.-von-Haunerschen-Kinderspital

Universität München

Lindwurmstraße 4

80336 München

\section{Erklärung zu Interessenkonflikten}

Die Autoren erklären, dass bei der Erstellung des Beitrags kein Interessenkonflikt vorlag. 